\title{
Primary Adrenal Insufficiency due to a Hypercoagulable State
}

\author{
Yasmine Elamir ${ }^{\mathrm{a}, \mathrm{c}}$, Louis Amorosa ${ }^{\mathrm{b}}$, William Grist ${ }^{\mathrm{a}}$
}

\begin{abstract}
To illustrate a case of primary adrenal insufficiency caused secondarily to eosinophilic granulomatosis with polyangiitis, case report and review of the literature are used. An example of primary adrenal hemorrhage due to a hypercoagulable state with a thorough discussion and review of the literature. Although rare a hypercoagulable state can lead to bilateral adrenal hemorrhages and subsequent primary adrenal insufficiency. Keeping this in mind can lead to prompter diagnosis and treatment.
\end{abstract}

Keywords: Adrenal insufficiency; Adrenal; Mhypercoagulable

\section{Introduction}

Primary adrenal insufficiency (AI) is generally caused by an autoimmune phenomenon but can occur secondary to adrenal hemorrhage $(\mathrm{AH})$ which can occur secondary to a hypercoagulable state. Here we present such a case where a man with eosinophilic granulomatosis with polyangiitis (EGPA) presents with bilateral AHs, presumably secondary to a hypercoagulable state leading ultimately to AI.

\section{Case Report}

Patient is a 59-year-old Caucasian male with a past medical history of EGPA, cellulitis, and colon resection for polyps who presented to the emergency department with dyspnea. A month prior to this admission, the patient had presented with abdominal pain after 2 weeks of failed outpatient cellulitis treatment with antibiotics. An abdominal CT at that time showed bilateral adrenal masses. During this admission, his complete blood count (CBC) revealed 47\% eosinophils and chest X-ray showed pulmonary infiltrates. A CT scan of the

Manuscript submitted May 28, 2018, accepted June 20, 2018

aJersey City Medical Center, Jersey City, NJ, USA

bRobert Wood Johnson University Hospital, Jersey City, NJ, USA

${ }^{\mathrm{c} C}$ Corresponding Author: Yasmine Elamir, Jersey City Medical Center, 355

Grand St., Jersey City, NJ 07302, USA. Email: yasmine.elamir@rwjbh.org

doi: https://doi.org/10.14740/jmc3100w chest revealed a pulmonary embolism with infarctions noted. Incidentally noted on the scan were bilateral $3-4 \mathrm{~cm}$ adrenal gland masses that were seen on the abdominal CT the previous admission. Previous abdominal imaging from 8 years ago during colon resection revealed no masses. During the hospital course his "cellulitis" was biopsied and revealed eosinophilic angiitis with granulomas. After confirming the diagnosis of EGPA, the patient was treated with steroids titrated to prednisone $40 \mathrm{mg}$ daily which resolved the lower extremity rash. The patient's pulmonary embolism was treated with a heparin drip while inpatient and the patient was discharged on oral anticoagulation. Over the course of next few months, the patient's steroids were decremented to $5 \mathrm{mg}$ of prednisone daily and symptoms of fatigue began to insidiously occur. The fatigue only improved when he was started on high dosing of hydrocortisone. Due to the patient's profound weakness that improved with steroid treatment, we performed a three day cosyntropin test (CST) because our patient was already on a prolonged course of prednisone. Cortisol level after adrenocorticotropic hormone (ACTH) stimulation should be higher than $18-20 \mathrm{mcg} / \mathrm{dL}$. We found the cortisol level to be $7 \mathrm{mcg} /$ $\mathrm{dL}$ on the first day, $6 \mathrm{mcg} / \mathrm{dL}$ on the second day and $6 \mathrm{mcg} /$ $\mathrm{dL}$ on the third day. The aldosterone level was also decreased at $5.9 \mathrm{ng} / \mathrm{dL}$. This confirmed our diagnosis of primary AI. The patient had been admitted again at a later date for shortness of breath, and a CT chest was obtained showing resolution of the bilateral adrenal masses. Presumptively the hypercoagulable state and subsequent adrenal infarctions were due to the inflammatory response from his eosinophilic vasculitis. This, combined with anticoagulation for pulmonary embolism, led to AHs resulting in primary AI.

\section{Discussion}

Primary AI is rarely caused by AH. Etiologies include, but are not limited to, sepsis, myocardial infarction, an underlying mass, malignancy, pheochromocytoma, trauma, meningococcemia (Waterhouse-Friderichsen syndrome) anticoagulation or a hypercoagulable state such as antiphospholipid syndrome (APS) [1]. However, it has not yet been reported in the literature as being caused by EGPA or a hyper-eosinophilic syndrome [2]. Our case was also interesting in the fact that primary AI was not apparent during his initial presentation. Likely, his symptoms were masked by the steroids which were given for his EGPA diagnosed on admission. It was not until the dose was decremented that the primary AI was investigated due to 
symptoms of worsening fatigue.

The association of hypercoagulable states and APS with the development of $\mathrm{AH}$ and $\mathrm{AI}$ are referenced frequently. There are also cases that reference plasmapharesis as the cause of hypercoagulability $[3,4]$. The mechanism for APS causing adrenal insufficiency has not yet been defined. One theory is that due to high levels of cholesterol in the adrenal glands, antiphospholipid antibodies are attracted, which leads to thrombosis and hemorrhage. Another theory is that due to the rich blood supply into the adrenal gland and slow transit of blood out of the adrenal glands, there is a predisposition to stasis and eventual adrenal thrombosis leading to subsequent adrenal hemorrhage, especially in a hypercoagulable state. This is due to the anatomy of the adrenal gland. Its venous system is limited with one central vein compared to an extensive arterial system that supplies blood to the adrenal gland [5]. The latter of the two theories could be a potential reason in which EGPA could attribute to AH causing AI.

EGPA is associated with a large amount of eosinophils in the blood and this alone can lead to increased risk of thromboembolism. This same risk would be applied to any disorder with marked eosinophilia including eosinophilic myeloproliferative disorders, idiopathic hypereosinophlic syndrome and eosinophilic vasculitis. The mechanism for which eosinophils cause hypercoagulability is unclear, but contributing factors likely include the following:

1) Initiation of the clotting cascade by tissue factor (TF) released during eosinophilic degranulation. Moosbauer et al demonstrates that eosinophils maintain a high TF expression during maturation, providing a main source of preformed TF in blood which might be relevant for the thombogenesis promoted by hypereosinophilic conditions [6].

2) Inhibition of vascular endothelial thrombomodulin (a potent anticoagulant) by major basic protein (MBP) an eosinophilic specific granule protein. It was proposed by Slungaard et al that these eosinophilic cationic proteins bind to anionic thrombomodulin, preventing its anticoagulant activities, including generation of natural anticoagulant activated protein C (APC) [7].

3) Activation of platelets by MBP and eosinophil peroxidase. Two of the four principal cationic proteins of the eosinophil granule were demonstrated by Rohrbach et al to be strong platelet agonists which would surely lead to a hypercoagulable state [8].

Thromboembolic disease is seen in many vasculitides. In a review article by Tomasson et al, it is shown that besides the vasculitis in and of itself being linked to thrombosis, patients with anti-neutrophil cytoplasmic antibodies (ANCA)associated vasculitis have been identified to explain the high occurrence of thrombosis. Our patient in the case above was also ANCA-positive. A better understanding of the association of thrombosis with inflammation in vasculitis could eventually lead to useful biomarkers and approaches to therapy that could potentially help prevent these types of catastrophes from happening in the future [9]. In a systemic retrospective study on 1,130 patients, it was noted that like granulomatosis with polyangiitis and microscopic polyangiitis, patients with EGPA had a higher frequency of venous thromboembolic events. This is in contrast to vasculitides like polyarteritis no- dosa, which is ANCA-negative. Of note, most of the thrombotic events that occurred in the study were close in temporal proximity to the time of diagnosing the vasculitis. This was also similar in our case as they were so close in proximity that the treatment of the EGPA masked the occurrence of the AH leading to AI. This suggests that thrombotic events associated with these types of vasculitis are tightly associated with disease activity [10].

Generally, both adrenal glands are affected when hypercoagulable states lead to AH. The AHs of both glands can be simultaneous or a few days apart [11]. This usually causes irreversible and complete adrenal failure [5]. Therefore, if one were to find a hemorrhage in only one adrenal gland it would be pivotal to repeat imaging in a few days to see if the other adrenal gland had hemorrhaged as well. When an acute bilateral adrenal hemorrhage happens, biochemical confirmation of hypo-adrenalism is needed to confirm the diagnosis of AI which includes a low cortisol level and an elevated ACTH. Of note if a patient has been chronically on steroids, an ACTH level will not be as helpful in the diagnosis as the level will not be accurate. Once a diagnosis is confirmed, treatment with corticosteroids should be started right away. Generally daily doses range between 150 and $200 \mathrm{mg}$ of hydrocortisone [12]. Patients will require lifelong steroid replacement.

It is important to discuss that the patient's AH was present on CT at the same time of the pulmonary embolismprior to treatment with anticoagulation. Likely both findings were caused by the hypercoagulable state of EGPA. This excludes the possibility that the patient's anticoagulation was the cause of the $\mathrm{AH}$. The separation of the events is imperative as anticoagulation is a cause of AH and therefore, AI. The fact that the adrenal masses disappeared later on CT also demonstrates that the masses were hemorrhages. From these findings, it can be concluded that the hypercoagulable state was the actual mechanism for the development of bilateral $\mathrm{AH}$ and subsequent $\mathrm{AI}$ in our case. This illustrates the importance of sequential imaging and to always consider hypercoagulable states as the etiology of hemorrhagic adrenal masses.

\section{Conclusions}

This is a rare example of where a hypercoagulable state led to a bilateral AH and subsequent primary AI. The patient's history of steroid use likely masked his symptoms for a long time which made our case unique. In addition, it illustrates the importance of sequentially seeing the occurrence and disappearance of the adrenal masses on CT to aid in accurately diagnosing $\mathrm{AH}$ and the likely etiology in this patient. It also shows that there must be a high index of suspicion for hypercoagulable states when looking for the etiology of hemorrhagic adrenal masses. This highlights the importance of proper evaluation of $\mathrm{AI}$ and use of formal CST to evaluate the etiology of fatigue. After reviewing the literature, we have not identified a similar case with EGPA, although we have found similar cases with APS and plasmapheresis causing a procoagulant impact. Our case illustrates the potential catastrophic complication of this 
condition.

\section{Disclosure}

The authors have no multiplicity of interest to disclose.

\section{Data}

All of the data for this case report were retrieved from the electronic heath record of Robert Wood Johnson University Hospital. All of the relevant data are presented in the case report.

\section{References}

1. Rao RH. Bilateral massive adrenal hemorrhage. Med Clin North Am. 1995;79(1):107-129.

2. Marti JL, Millet J, Sosa JA, Roman SA, Carling T, Udelsman R. Spontaneous adrenal hemorrhage with associated masses: etiology and management in 6 cases and a review of 133 reported cases. World J Surg. 2012;36(1):75-82.

3. Surov S, Ovsepyan R, Vysochin I, Kobzeva E, Khvatov V, Panteleev M, Vuimo T. Procoagulant impact of the plasmapheresis procedure on coagulation state of collected plasma. Blood Transfus. 2015;13(4):651-655.

4. Saleem N, Khan M, Parveen S, Balavenkatraman A. Bilateral adrenal haemorrhage: a cause of haemodynamic collapse in heparin-induced thrombocytopaenia. BMJ Case Rep. 2016;2016.

5. Ramon I, Mathian A, Bachelot A, Hervier B, Haroche J, Boutin-Le Thi Huong D, Costedoat-Chalumeau N, et al. Primary adrenal insufficiency due to bilateral adrenal hemorrhage-adrenal infarction in the antiphospholipid syndrome: long-term outcome of 16 patients. J Clin Endocrinol Metab. 2013;98(8):3179-3189.

6. Moosbauer C, Morgenstern E, Cuvelier SL, Manukyan D, Bidzhekov K, Albrecht S, Lohse P, et al. Eosinophils are a major intravascular location for tissue factor storage and exposure. Blood. 2007;109(3):995-1002.

7. Slungaard A, Vercellotti GM, Tran T, Gleich GJ, Key NS. Eosinophil cationic granule proteins impair thrombomodulin function. A potential mechanism for thromboembolism in hypereosinophilic heart disease. J Clin Invest. 1993;91(4):1721-1730.

8. Rohrbach MS, Wheatley CL, Slifman NR, Gleich GJ. Activation of platelets by eosinophil granule proteins. J Exp Med. 1990;172(4):1271-1274.

9. Tomasson G, Monach PA, Merkel PA. Thromboembolic disease in vasculitis. Curr Opin Rheumatol. 2009;21(1):41-46.

10. Allenbach Y, Seror R, Pagnoux C, Teixeira L, Guilpain P, Guillevin L, French Vasculitis Study G. High frequency of venous thromboembolic events in Churg-Strauss syndrome, Wegener's granulomatosis and microscopic polyangiitis but not polyarteritis nodosa: a systematic retrospective study on 1130 patients. Ann Rheum Dis. 2009;68(4):564-567.

11. Berneis K, Buitrago-Tellez C, Muller B, Keller U, Tsakiris DA. Antiphospholipid syndrome and endocrine damage: why bilateral adrenal thrombosis? Eur J Haematol. 2003;71(4):299-302.

12. Shenker Y, Skatrud JB. Adrenal insufficiency in critically ill patients. Am J Respir Crit Care Med. 2001;163(7):15201523. 